\title{
RESULTS OF SELFMONITORING ON GLUCOMETER SYSTEMS ADVANCE AND OPTIUM IN DAILY ROUTINE
}

\author{
Rudolf Chlup a,b, Martin Payne ${ }^{\mathrm{c}}$, Jana Zapletalovád, Stanislav Komenda ${ }^{\mathrm{d}}$, Blanka Doubravováe, \\ Martina Řezníčkováa , Ludmila Chlupováf, Pavel Sečkar̆g
}

a Institute of Physiology, Faculty of Medicine, Palacký University, Olomouc

${ }^{b}$ IInd Dept. of Medicine, Faculty of Medicine, Palacký University \& Teaching Hospital, Olomouc

c Hypoguard Limited, Woodbridge, United Kingdom

d Department of Biometrics, Faculty of Medicine, Palacký University, Olomouc

e Institute of Neurology and Geriatrics, Moravský Beroun, Czech Republic

Department of Special Education, Pedagogical Faculty, Palacký University, Olomouc

$g$ Department of Health Insurance, Teaching Hospital Olomouc, Czech Republic

Received: April 10, 2005; Accepted (with revisions): May 18, 2005

Key words: Glucometer Advance/Glucometer Optium/Selfmonitoring/Precision/Software Glucobalance/Software PCLink

The aim of this prospective clinical study was to compare the results of B-glucose estimations performed simultaneously on glucometer Advance (with Micro-draw strips) and Optium (G3 strips) by lay healthy volunteers under non-standardized conditions of everyday life, to assess the difficulties dealing with lay - handling of these systems and to demonstrate the possibilities of the software Glucobalance (Hypoguard) and PC-Link (Medisense/Abbott) for the analysis of selfmonitoring. In the course of 5 days, a total of 721 pairs of measurements were carried out on 10 pairs of glucometer Advance and Optium by 10 healthy volunteers aged 16-40 years. The data transfer of all values into computer from glucometer Advance using the Glucobalance software and from glucometer Optium using the PC-Link was carried out to determine the results. The correlation of B-glucose measured on the glucometer Advance and Optium was strong $(\mathrm{r}=0.73)$. Glucometer Advance brings values about $0.21 \pm 0.06 \mathrm{mmol} / 1$ lower than glucometer Optium. The average difference found within each pairs of glucometers Advance - Optium varied. Nevertheless, these differences are acceptable for routine selfmonitoring. The handling of glucometer Advance is not difficult for lay persons. The Glucobalance software simplifies the result evaluation by each tested person. Even though there are some advantages in comparison with the PC-Link, it should be further developed.

\section{INTRODUCTION}

Blood glucose selfmonitoring has become a prerequisite in the successful treatment of both type 1 and type 2 diabetes mellitus $3,4,13,15,22$. In this regard different types of glucometers are marketed to cover increasing demands ${ }^{11,24}$. The Glucometer Advance (Hypoguard, Great Britain) and the recently developed new generation of capillary strips (Micro-draw) comprise an approved B-glucose selfmonitoring system (GSS) which is ready to use in many countries. Glucometer Optium (Medisense/Abbott) and G 3 strips are already known to produce acceptable results from several years experience ${ }^{14}$.

The aim of this prospective clinical study was:

1. to compare the results of B-glucose estimations performed simultaneously on glucometer Advance (with Micro-draw strips) and Optium (G3 strips) by lay healthy volunteers under the non-standardized conditions of everyday life;

2. to assess the difficulties dealing with lay - handling of these systems;
3. to demonstrate the possibilities of the software Glucobalance (Hypoguard) and PC-Link (Medisense/Abbott) for the analysis of selfmonitoring.

\section{METHODS}

\section{Tested persons}

Ten healthy volunteers aged between 16 and 40 years (students, nurses, laboratory assistants) having no personal experience with GSS Advance or Optium entered the study. Informed consent was obtained from all of them. Everyone received one glucometer Advance, one glucometer Optium and two packages of strips per glucometer (Table 1). All volunteers were given a 30 min training in selfmonitoring. None of the glucometers with the exception of Optium No 6 had been used before.

\section{Study design}

All measurements were performed in July 2003 in the course of one week, usually at home, at work, in a hospi- 
tal ward or on a trip. The weather conditions were good, temperatures mostly in between $15-30{ }^{\circ} \mathrm{C}$.

Each volunteer was encouraged to perform B-glucose selfmonitoring at breakfast and at dinner, according to the following 7-point schedule: before the meal (time 0) and $15,30,45,60,90$ and 120 min following the start of the meal. In this way, everyone was performing 14 estimations per day.

In selfmonitoring, capillary blood was obtained from a finger by means of a lancing device; the first drop was put on a strip G3 (Optium) and, immediately afterwards, the second drop was put on a Micro-draw strip (Advance). The volunteer registered all results read on the displays of both glucometers in the protocol.

At the end of the one-week test period each volunteer was asked to describe the positive and the negative features of individual tested systems.

\section{Statistical analysis}

The data were transferred by means of respective software (Glucobalance or PC-Link) from the glucometers to a PC.

The program MS Excel and the statistical package SPSS, v. 10.1, were used for analyzing the results: t-test for matched observations, Correlation Analyses a Analyses of Variance were applied to evaluate the data. The level of significance $\mathrm{p}<0.05$ was taken.

\section{RESULTS}

In the course of 5 days, a total of 721 pairs of measurements were carried out on 10 pairs of glucometer Advance and Optium by 10 volunteers. One measurement showed an extreme BG value of $42.8 \mathrm{mmo} / 1$; as this value exceeded the declared range of glucometer (1.1-33.3 $\mathrm{mmol} / \mathrm{l}$ ), the pair concerned was not considered for statistical analysis.

For the results of measurements from individual glucometers see Table 2. Occasionally there were some high (>14.0 mmol/1) or low (<3.3 mmol/1) B-glucose values measured on both glucometers Advance and Optium. There is no explanation for these unusual values.

There was a strong correlation between the B-glucose values measured on all glucometers Advance and Optium ( $r=0.73$, see Fig. 1). However, the correlation coefficients of the individual pairs of glucometers differed $(r=0.58$ to $r=0.91$, see Table 3).

For the frequency of B-glucose differences in all 10 pairs of glucometers (Advance - Optium) see the histogram in Fig. 2. The majority of all differences (96,1\%) was within $\pm 2.25 \mathrm{mmol} / 1$. Extreme differences were registered only rarely (Table 4).

For the results of statistical analysis see Table 5: the B-glucose values on the Advance glucometers were lower about $0.21 \pm 0.06 \mathrm{mmol} / 1(\mathrm{x} \pm \mathrm{SE})$, i.e. $2.998 \%$, than the values measured on the glucometer Optium. This difference did not depend on the absolute B-glucose concentration (Fig. 3).

Table 1. Characteristics of tested glucometer systems Advance and Optium

\begin{tabular}{|c|c|c|c|c|}
\hline No & $\begin{array}{c}\text { ADVANCE } \\
\text { serial number }\end{array}$ & $\begin{array}{c}\text { Strips } \\
\text { Batch No }\end{array}$ & $\begin{array}{c}\text { OPTIUM } \\
\text { serial number }\end{array}$ & $\begin{array}{c}\text { Strips } \\
\text { Batch No }\end{array}$ \\
\hline 1 & SN 040001227 & 6004 & $\begin{array}{c}\text { QA0849-4292 } \\
\text { P/N 120-203-08 }\end{array}$ & 51322 \\
\hline 2 & SN 040002678 & 6004 & $\begin{array}{c}\text { QA2132-1992 } \\
\text { P/N 120-503-08 }\end{array}$ & 51322 \\
\hline 3 & SN 040002667 & 6004 & $\begin{array}{l}\text { QA2501-1466 } \\
120-503-08 \text { C4 }\end{array}$ & 51322 \\
\hline 4 & SN 040002688 & 6004 & $\begin{array}{c}\text { QA0849-4532 } \\
\text { P/N 120-203-08 }\end{array}$ & 51322 \\
\hline 5 & SN 040002685 & 6004 & $\begin{array}{l}\text { QA2501-3595 } \\
120-503-08 \text { C4 }\end{array}$ & 51322 \\
\hline 6 & SN 040002689 & 6004 & $\begin{array}{c}\text { QA0369-2065 } \\
\text { P/N 120-230-08 }\end{array}$ & 51322 \\
\hline 7 & SN 040002704 & 6004 & $\begin{array}{l}\text { QA2501-3261 } \\
120-503-08 \text { C4 }\end{array}$ & 51322 \\
\hline 8 & SN 040002694 & 6004 & $\begin{array}{c}\text { QA2501-1461 } \\
120-503-08 \text { C4 }\end{array}$ & 51322 \\
\hline 9 & SN 040002697 & 6004 & $\begin{array}{c}\text { QA2202-3555 } \\
\text { P/N 120-503-08 }\end{array}$ & 51322 \\
\hline 10 & SN 040002666 & 6004 & $\begin{array}{c}\text { QA2202-1771 } \\
\text { P/N120-503-08 }\end{array}$ & 51322 \\
\hline
\end{tabular}


Table 2. Results of measurements from individual pairs of glucometers Advance (A) and Optium (O)

\begin{tabular}{|ll|r|r|r|}
\hline \multicolumn{1}{|c|}{ PROBAND } & ADVANCE & OPTIUM & \multicolumn{1}{l|}{ A-O } \\
\hline 1 & $\mathrm{~N}$ & 97 & 97 & 97 \\
& Minimum & 3.3 & 4.00 & -3.09 \\
& Maximum & 15.1 & 13.00 & 5.16 \\
& Median & 8.400 & 7.5600 & .8300 \\
& Mean & 8.493 & 7.5919 & .9009 \\
& Std. Deviation & 2.561 & 2.2967 & 1.5508 \\
\hline 2 & $\mathrm{~N}$ & 104 & 104 & 104 \\
& Minimum & 1.4 & 3.56 & -4.79 \\
& Maximum & 10.0 & 10.89 & 2.56 \\
& Median & 5.600 & 5.9700 & -.5900 \\
& Mean & 5.565 & 6.0994 & -.5340 \\
& Std. Deviation & 1.255 & 1.0781 & 1.0807 \\
\hline 3 & $\mathrm{~N}$ & 56 & 56 & 56 \\
& Minimum & 3.6 & 4.61 & -1.62 \\
& Maximum & 10.0 & 9.78 & 2.19 \\
& Median & 6.850 & 5.8900 & .7300 \\
& Mean & 6.827 & 6.1836 & .6432 \\
& Std. Deviation & 1.127 & 1.2812 & .9365 \\
\hline 4 & $\mathrm{~N}$ & 55 & 55 & 55 \\
& Minimum & 1.9 & 3.44 & -5.88 \\
& Maximum & 12.3 & 12.50 & .69 \\
& Median & 5.300 & 7.2200 & -1.6800 \\
& Mean & 5.338 & 7.1251 & -1.7869 \\
& Std. Deviation & 2.258 & 2.1265 & 1.4753 \\
\hline 5 & N & 64 & 64 & 64 \\
& Minimum & 1.2 & 3.72 & -5.67 \\
& Maximum & 21.6 & 13.39 & 9.82 \\
& Median & 6.050 & 6.9400 & -1.1300 \\
& Mean & 6.470 & 7.4472 & -.9769 \\
& Std. Deviation & 3.068 & 1.8851 & 2.1749 \\
\hline & & & & \\
\hline
\end{tabular}

\begin{tabular}{|c|c|c|c|c|}
\hline & PROBAND & ADVANCE & OPTIUM & $\mathrm{A}-\mathrm{O}$ \\
\hline 6 & $\mathrm{~N}$ & 100 & 100 & 100 \\
\hline & Minimum & 3.0 & 3.17 & -3.47 \\
\hline & Maximum & 13.9 & 14.50 & 1.83 \\
\hline & Median & 6.50 & 6.9150 & -.5600 \\
\hline & Mean & 6.96 & 7.5274 & -.5684 \\
\hline & Std. Deviation & 2.00 & 2.0497 & .8567 \\
\hline 7 & $\mathrm{~N}$ & 47 & 47 & 47 \\
\hline & Minimum & 1.1 & 4.06 & -4.70 \\
\hline & Maximum & 11.3 & 10.83 & 1.13 \\
\hline & Median & 5.00 & 6.1700 & -1.0800 \\
\hline & Mean & 5.31 & 6.6472 & -1.3366 \\
\hline & Std. Deviation & 1.63 & 1.6876 & 1.1886 \\
\hline 8 & $\mathrm{~N}$ & 62 & 62 & 62 \\
\hline & Minimum & 2.5 & 4.33 & -3.39 \\
\hline & Maximum & 11.7 & 10.83 & 3.93 \\
\hline & Median & 6.80 & 6.6150 & -1900 \\
\hline & Mean & 6.70 & 6.6485 & 0482 \\
\hline & Std. Deviation & 1.65 & 1.4717 & 1.1254 \\
\hline 9 & $\mathrm{~N}$ & 78 & 78 & 78 \\
\hline & Minimum & 3.7 & 3.28 & -1.63 \\
\hline & Maximum & 18.8 & 12.39 & 8.02 \\
\hline & Median & 6.45 & 6.5600 & .1150 \\
\hline & Mean & 7.04 & 6.7637 & 2760 \\
\hline & Std. Deviation & 2.34 & 1.9786 & 1.3998 \\
\hline 10 & $\mathrm{~N}$ & 57 & 57 & 57 \\
\hline & Minimum & 3.9 & 3.67 & -1.34 \\
\hline & Maximum & 9.0 & 9.39 & 2.76 \\
\hline & Median & 6.50 & 5.5600 & .6800 \\
\hline & Mean & 6.66 & 5.9556 & .7058 \\
\hline & Std. Deviation & 1.30 & 1.5020 & .9407 \\
\hline Total & $\mathrm{N}$ & 720 & 720 & 720 \\
\hline & Minimum & 1.1 & 3.17 & -5.88 \\
\hline & Maximum & 21.6 & 14.50 & 9.82 \\
\hline & Median & 6.30 & 6.5600 & -2800 \\
\hline & Mean & 6.64 & 6.8471 & -2053 \\
\hline & Std. Deviation & 2.23 & 1.8838 & 1.5462 \\
\hline
\end{tabular}

The results of analysis of variance of the differences of each glucometer is shown in Table 6: mean B-glucose differences within individual pairs of glucometers are often significantly different.

The volunteers agreed that the usage of both types of glucometers was simple. The glucometer Advance was appreciated for its small, handy size, the option of going through the memory results forwards and backwards and the very small amount of blood needed for one measurement. On the other hand, the large strip packages didn't fit into the box together with the glucometer and this was seen as a disadvantage.
The data transfer of all values into computer from glucometer Advance using the Glucobalance software and from glucometer Optium using the PC-link was carried out by a professional. The Glucobalance and PC-link enabled as to see the results of an individual volunteer separately. One of the advantages of the Glucobalance is the ability to edit histograms (Fig. 4), pie charts (Fig. 5) and statistics (Fig. 6) for individually set day time periods. The modal day (Fig. 7) and time trends (Fig. 8) are similar to those of the PC Link. None of the programs enables to compare the data interindividually. None of the programs comes in a Czech version. 
Table 3. Correlations between the B-glucose values measured on individual pairs of glucometers Advance (A) - Optium (O)

\begin{tabular}{|c|c|c|c|c|c|}
\hline & $\mathrm{O} 1$ & $\mathrm{O} 2$ & $\mathrm{O} 3$ & $\mathrm{O} 4$ & $\mathrm{O} 5$ \\
\hline Al Pearson Correlation & $.801 *$ & & & & \\
Sig. (2-tailed) & .000 & & & & \\
N & 97 & & & & \\
\hline A2 Pearson Correlation & & $.580^{*}$ & & & \\
$\quad$ Sig. (2-tailed) & & .000 & & & \\
N & & 104 & & & \\
\hline A3 Pearson Correlation & & & $.704^{*}$ & & \\
$\quad \begin{array}{l}\text { Sig. (2-tailed) } \\
\text { N }\end{array}$ & & & .000 & & \\
\hline A4 Pearson Correlation & & & & $.775^{*}$ & \\
$\quad$ Sig. (2-tailed) & & & & .000 & \\
N & & & & 55 & \\
\hline A5 Pearson Correlation & & & & & $.712^{*}$ \\
Sig. (2-tailed) & & & & & .000 \\
N & & & & & 64 \\
\hline
\end{tabular}

\begin{tabular}{|l|c|c|c|c|c|}
\hline & O6 & O7 & O8 & O9 & O10 \\
\hline A6 Pearson Correlation & $.911^{*}$ & & & & \\
Sig. (2-tailed) & .000 & & & & \\
N & 100 & & & & \\
\hline A7 Pearson Correlation & & $.744^{*}$ & & & \\
Sig. (2-tailed) & & .000 & & & \\
N & & 47 & & & \\
\hline A8 Pearson Correlation & & & $.746^{*}$ & & \\
Sig. (2-tailed) & & & .000 & & \\
N & & & 62 & & \\
\hline A9 Pearson Correlation & & & & $.802^{*}$ & \\
Sig. (2-tailed) & & & & .000 & \\
N & & & & 78 & \\
\hline A10 Pearson Correlation & & & & & $.784^{*}$ \\
Sig. (2-tailed) & & & & & .000 \\
N & & & & & 57 \\
\hline
\end{tabular}

* Correlation is significant at the 0.01 level (2-tailed).

Table 4. Frequency table: B-glucose difference Advance - Optium

\begin{tabular}{|r|r|r|c|}
\hline $\begin{array}{c}\text { Difference } \\
{[\mathrm{mmol} / \mathrm{l}]}\end{array}$ & Frequency & Percent & $\begin{array}{c}\text { Cumula- } \\
\text { tive } \\
\text { Percent }\end{array}$ \\
\hline Valid $-6.25<=\mathrm{x}<-5.75$ & 1 & .1 & .1 \\
\hline$-5.75<=\mathrm{x}<-5.25$ & 3 & .4 & .6 \\
\hline$-5.25<=\mathrm{x}<-4.75$ & 2 & .3 & .8 \\
\hline$-4.75<=\mathrm{x}<-4.25$ & 5 & .7 & 1.5 \\
\hline$-4.25<=\mathrm{x}<-3.75$ & 2 & .3 & 1.8 \\
\hline$-3.75<=\mathrm{x}<-3.25$ & 5 & .7 & 2.5 \\
\hline$-3.25<=\mathrm{x}<-2.75$ & 11 & 1.5 & 4.0 \\
\hline$-2.75<=\mathrm{x}<-2.25$ & 18 & 2.5 & 6.5 \\
\hline$-2.25<=\mathrm{x}<-1.75$ & 38 & 5.3 & 11.8 \\
\hline$-1.75<=\mathrm{x}<-1.25$ & 51 & 7.1 & 18.9 \\
\hline$-1.25<=\mathrm{x}<-0.75$ & 110 & 15.3 & 34.2 \\
\hline$-0.75<=\mathrm{x}<-0.25$ & 123 & 17.1 & 51.3 \\
\hline$-0.25<=\mathrm{x}<0.25$ & 115 & 16.0 & 67.2 \\
\hline $0.25<=\mathrm{x}<0.75$ & 72 & 10.0 & 77.2 \\
\hline $0.75<=\mathrm{x}<1.25$ & 63 & 8.8 & 86.0 \\
\hline $1.25<=\mathrm{x}<1.75$ & 40 & 5.6 & 91.5 \\
\hline $1.75<=\mathrm{x}<2.25$ & 33 & 4.6 & 96.1 \\
\hline
\end{tabular}

\begin{tabular}{|c|c|c|c|}
\hline $\begin{array}{l}\text { Difference } \\
{[\mathrm{mmol} / \mathrm{l}]}\end{array}$ & Frequency & Percent & $\begin{array}{l}\text { Cumula- } \\
\text { tive } \\
\text { Percent }\end{array}$ \\
\hline $2.25<=\mathrm{x}<2.75$ & 12 & 1.7 & 97.8 \\
\hline $2.75<=x<3.25$ & 4 & .6 & 98.3 \\
\hline $3.25<=\mathrm{x}<3.75$ & 3 & .4 & 98.8 \\
\hline $3.75<=x<5.25$ & 2 & .3 & 99.0 \\
\hline $4.25<=x<4.75$ & 1 & .1 & 99.2 \\
\hline $4.75<=x<5.25$ & 3 & .4 & 99.6 \\
\hline $5.25<=x<5.75$ & 0 & .0 & 99.6 \\
\hline $5.75<=x<6.25$ & 1 & .1 & 99.7 \\
\hline $6.25<=x<6.75$ & 0 & .0 & 99.7 \\
\hline $6.75<=x<7.25$ & 0 & .0 & 99.7 \\
\hline $7.25<=\mathrm{x}<7.75$ & 0 & .0 & 99.7 \\
\hline $7.75<=x<8.25$ & 1 & .1 & 99.9 \\
\hline $8.25<=x<8.75$ & 0 & .0 & 99.9 \\
\hline $8.75<=x<9.25$ & 0 & .0 & 99.9 \\
\hline $9.25<=x<9.75$ & 0 & .0 & 99.9 \\
\hline $9.75<=x<10.25$ & 1 & .1 & 100.0 \\
\hline Total & 720 & 100.0 & \\
\hline
\end{tabular}


Table 5. Statistical evaluation of the B-glucose difference Advance - Optium (all results)

One-Sample Statistics

\begin{tabular}{|c|c|c|c|c|}
\hline & $\mathrm{N}$ & Mean & Std. Deviation & Std. Error Mean \\
\hline A_O Difference & 720 & -.205 & 1.5461 & .0576 \\
\hline
\end{tabular}

One-Sample Test

\begin{tabular}{|c|c|c|c|c|c|c|}
\hline & \multicolumn{6}{|c|}{ Test Value $=0$} \\
\hline & \multirow[t]{2}{*}{$\mathrm{t}$} & \multirow[t]{2}{*}{ df } & \multirow{2}{*}{$\begin{array}{c}\text { Sig. } \\
\text { (2-tailed) }\end{array}$} & \multirow{2}{*}{$\begin{array}{c}\text { Mean } \\
\text { Difference }\end{array}$} & \multicolumn{2}{|c|}{$\begin{array}{l}95 \% \text { Confidence } \\
\text { Interval of the Difference }\end{array}$} \\
\hline & & & & & Lower & Upper \\
\hline A_O Difference & -3.562 & 719 & .0004 & -.205 & -.318 & -.092 \\
\hline
\end{tabular}

Table 6. Statistical evaluation of the B-glucose difference Advance - Optium (ANOVA - multiple comparisons of individual patients i.e. pairs of glucometers)

\begin{tabular}{|c|c|c|c|c|c|c|}
\hline \multirow{2}{*}{ (1) PROBAND } & \multirow{2}{*}{ (J) PROBAND } & \multirow{2}{*}{$\begin{array}{c}\text { Mean } \\
\text { Difference } \\
(\mathrm{I}-\mathrm{J})\end{array}$} & \multirow{2}{*}{$\begin{array}{l}\text { Std. } \\
\text { Error }\end{array}$} & \multirow{2}{*}{ Sig. } & \multicolumn{2}{|c|}{$\begin{array}{l}\text { 95\% Confidence } \\
\text { Interval }\end{array}$} \\
\hline & & & & & $\begin{array}{l}\text { Lower } \\
\text { Bound }\end{array}$ & $\begin{array}{l}\text { Upper } \\
\text { Bound }\end{array}$ \\
\hline \multirow[t]{9}{*}{1} & 2 & $1.435^{*}$ & .186 & .000 & 1.070 & 1.800 \\
\hline & 3 & .258 & .221 & .244 & -.176 & .691 \\
\hline & 4 & $2.688^{*}$ & .222 & .000 & 2.252 & 3.124 \\
\hline & 5 & $1.878^{*}$ & .212 & .000 & 1.462 & 2.294 \\
\hline & 6 & $1.469 *$ & .188 & .000 & 1.101 & 1.838 \\
\hline & 7 & $2.238 *$ & .234 & .000 & 1.778 & 2.697 \\
\hline & 8 & $.853^{*}$ & .214 & .000 & .432 & 1.273 \\
\hline & 9 & $.625^{*}$ & .200 & .002 & .232 & 1.018 \\
\hline & 10 & .195 & .220 & .375 & -.236 & .626 \\
\hline \multirow[t]{8}{*}{2} & 3 & $-1.177 *$ & .218 & .000 & -1.606 & -.749 \\
\hline & 4 & $1.253^{*}$ & .219 & .000 & .822 & 1.684 \\
\hline & 5 & $.443^{*}$ & .209 & .035 & .032 & .853 \\
\hline & 6 & .034 & .184 & .852 & -.328 & .396 \\
\hline & 7 & $.803^{*}$ & .231 & .001 & .348 & 1.257 \\
\hline & 8 & $-.582 *$ & .211 & .006 & -.997 & -.168 \\
\hline & 9 & $-.810^{*}$ & .197 & .000 & -1.197 & -.423 \\
\hline & 10 & $-1.240^{*}$ & .217 & .000 & -1.666 & -.814 \\
\hline
\end{tabular}

\footnotetext{
* The mean difference is significant at the .05 level.
} 
Table 6 cont.

\begin{tabular}{|c|c|c|c|c|c|c|}
\hline \multirow{2}{*}{ (1) PROBAND } & \multirow{2}{*}{ (J) PROBAND } & \multirow{2}{*}{$\begin{array}{c}\text { Mean } \\
\text { Difference } \\
(\mathrm{I}-\mathrm{J})\end{array}$} & \multirow{2}{*}{$\begin{array}{l}\text { Std. } \\
\text { Error }\end{array}$} & \multirow{2}{*}{ Sig. } & \multicolumn{2}{|c|}{$\begin{array}{l}\text { 95\% Confidence } \\
\text { Interval }\end{array}$} \\
\hline & & & & & $\begin{array}{l}\text { Lower } \\
\text { Bound }\end{array}$ & $\begin{array}{l}\text { Upper } \\
\text { Bound }\end{array}$ \\
\hline \multirow[t]{7}{*}{3} & 4 & $2.430^{*}$ & .250 & .000 & 1.940 & 2.921 \\
\hline & 5 & $1.620^{*}$ & .241 & .000 & 1.147 & 2.093 \\
\hline & 6 & $1.212 *$ & .220 & .000 & .780 & 1.643 \\
\hline & 7 & $1.980^{*}$ & .260 & .000 & 1.469 & 2.491 \\
\hline & 8 & $.595^{*}$ & .243 & .014 & .119 & 1.071 \\
\hline & 9 & .367 & .231 & .112 & -.085 & .820 \\
\hline & 10 & -.063 & .248 & .801 & -.549 & .424 \\
\hline \multirow[t]{6}{*}{4} & 5 & $-.810 *$ & .242 & .001 & -1.285 & -.335 \\
\hline & 6 & $-1.219 *$ & .221 & .000 & -1.652 & -.785 \\
\hline & 7 & -.450 & .261 & .085 & -.964 & .063 \\
\hline & 8 & $-1.835^{*}$ & .244 & .000 & -2.314 & -1.356 \\
\hline & 9 & $-2.063^{*}$ & .232 & .000 & -2.518 & -1.608 \\
\hline & 10 & $-2.493^{*}$ & .249 & .000 & -2.981 & -2.004 \\
\hline \multirow[t]{5}{*}{5} & 6 & -.408 & .211 & .053 & -.822 & .005 \\
\hline & 7 & .360 & .253 & .155 & -.137 & .856 \\
\hline & 8 & $-1.025^{*}$ & .235 & .000 & -1.486 & -.565 \\
\hline & 9 & $-1.253^{*}$ & .222 & .000 & -1.689 & -.817 \\
\hline & 10 & $-1.683^{*}$ & .240 & .000 & -2.153 & -1.212 \\
\hline \multirow[t]{4}{*}{6} & 7 & $.768^{*}$ & .233 & .001 & .311 & 1.225 \\
\hline & 8 & $-.617 *$ & .213 & .004 & -1.034 & -.199 \\
\hline & 9 & $-.844^{*}$ & .199 & .000 & -1.235 & -.454 \\
\hline & 10 & $-1.274^{*}$ & .218 & .000 & -1.703 & -.845 \\
\hline \multirow[t]{3}{*}{7} & 8 & $-1.385^{*}$ & .255 & .000 & -1.885 & -.885 \\
\hline & 9 & $-1.613 *$ & .243 & .000 & -2.090 & -1.135 \\
\hline & 10 & $-2.042 *$ & .259 & .000 & -2.552 & -1.533 \\
\hline \multirow[t]{2}{*}{8} & 9 & -.228 & .224 & .309 & -.668 & .212 \\
\hline & 10 & $-.658 *$ & .242 & .007 & -1.132 & -.183 \\
\hline 9 & 10 & -.430 & .229 & .061 & -.880 & .021 \\
\hline
\end{tabular}

* The mean difference is significant at the .05 level. 


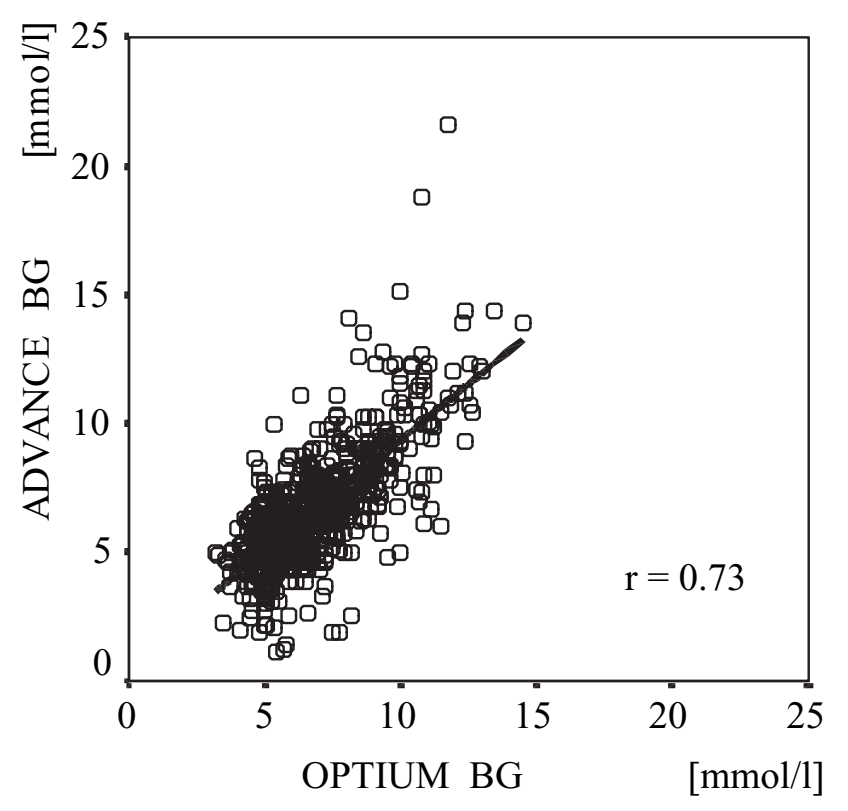

Fig. 1. Correlation between the B-glucose values measured on all glucometers Advance and Optium

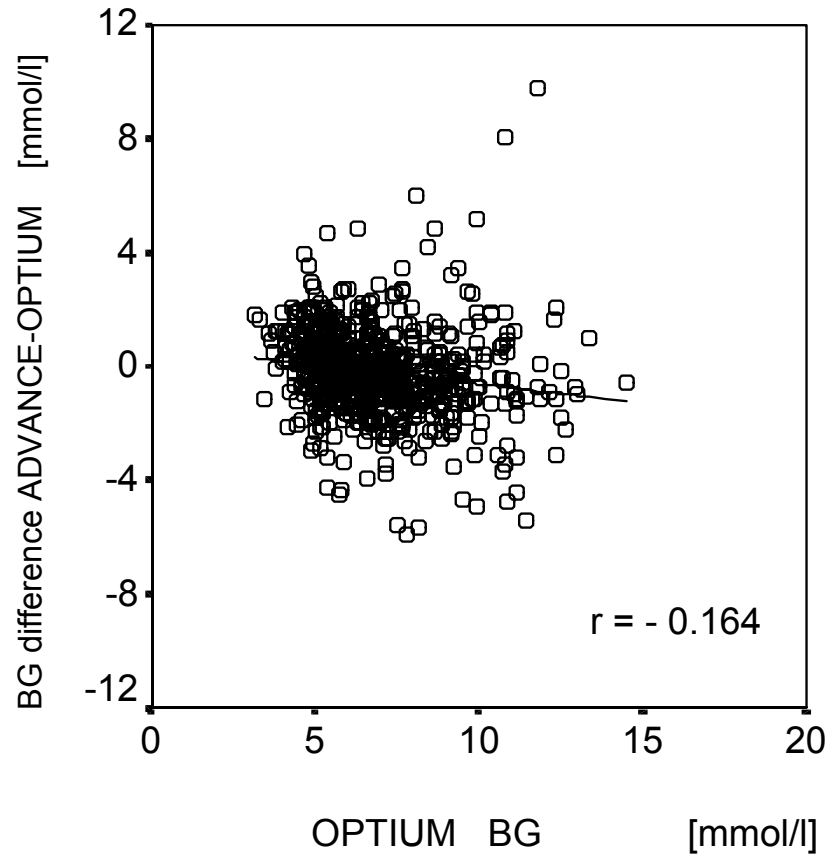

Fig. 3. Correlation between the B-glucose dfference Advance - Optium and the B-glucose value measured on glucometer Optium

\section{Histogram: difference ADVANCE-OPTIUM}

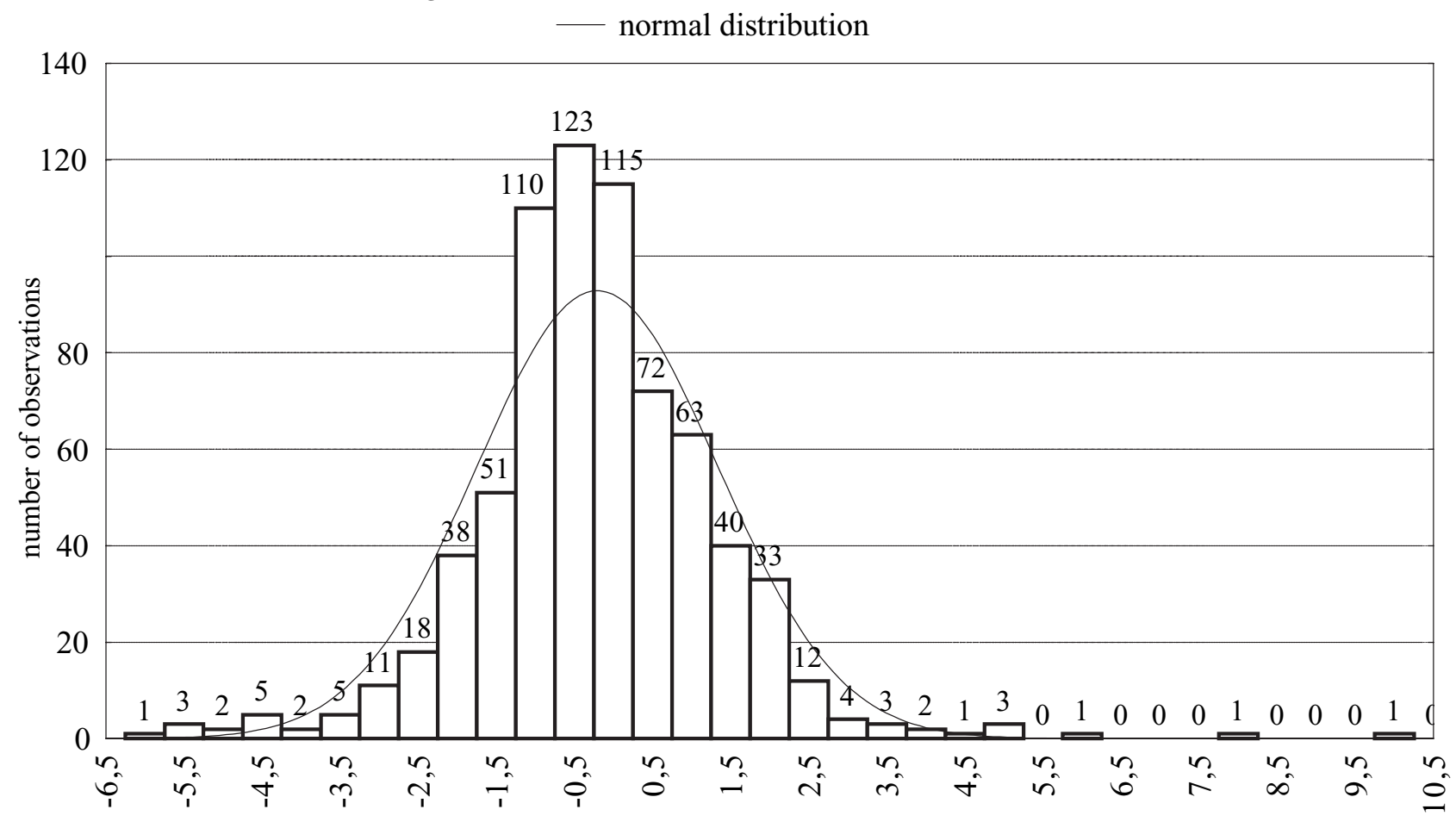

$[\mathrm{mmol} / \mathrm{l}]$

Fig. 2. Histogram: frequency (number of observations) of individual B-glucose differences Advance - Optium; total number of comparisons $n=720$ 


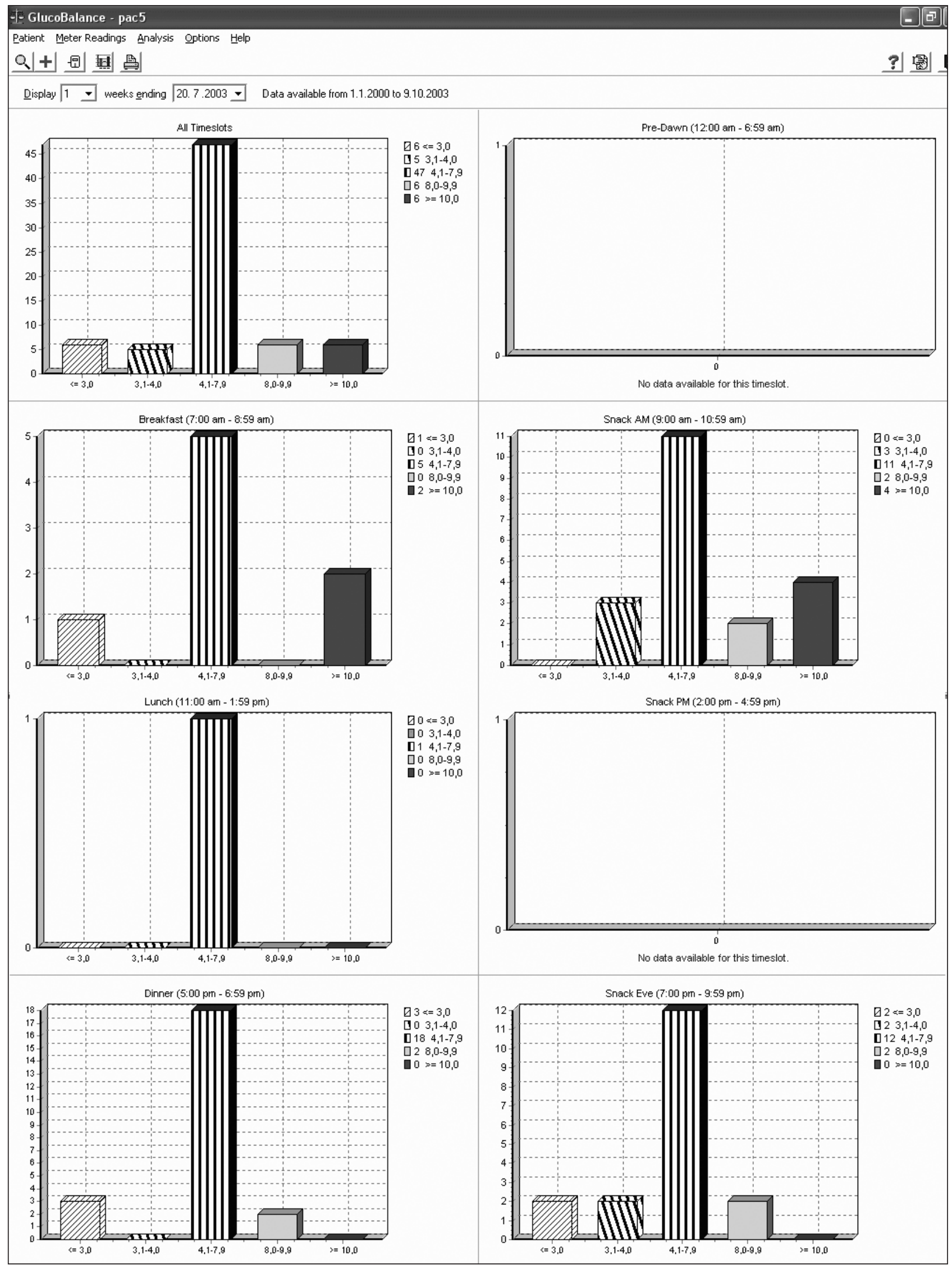

Fig. 4. Histograms of B-glucose values from the defined day time periods in one tested person (GSS Advance, software Glucobalance) 


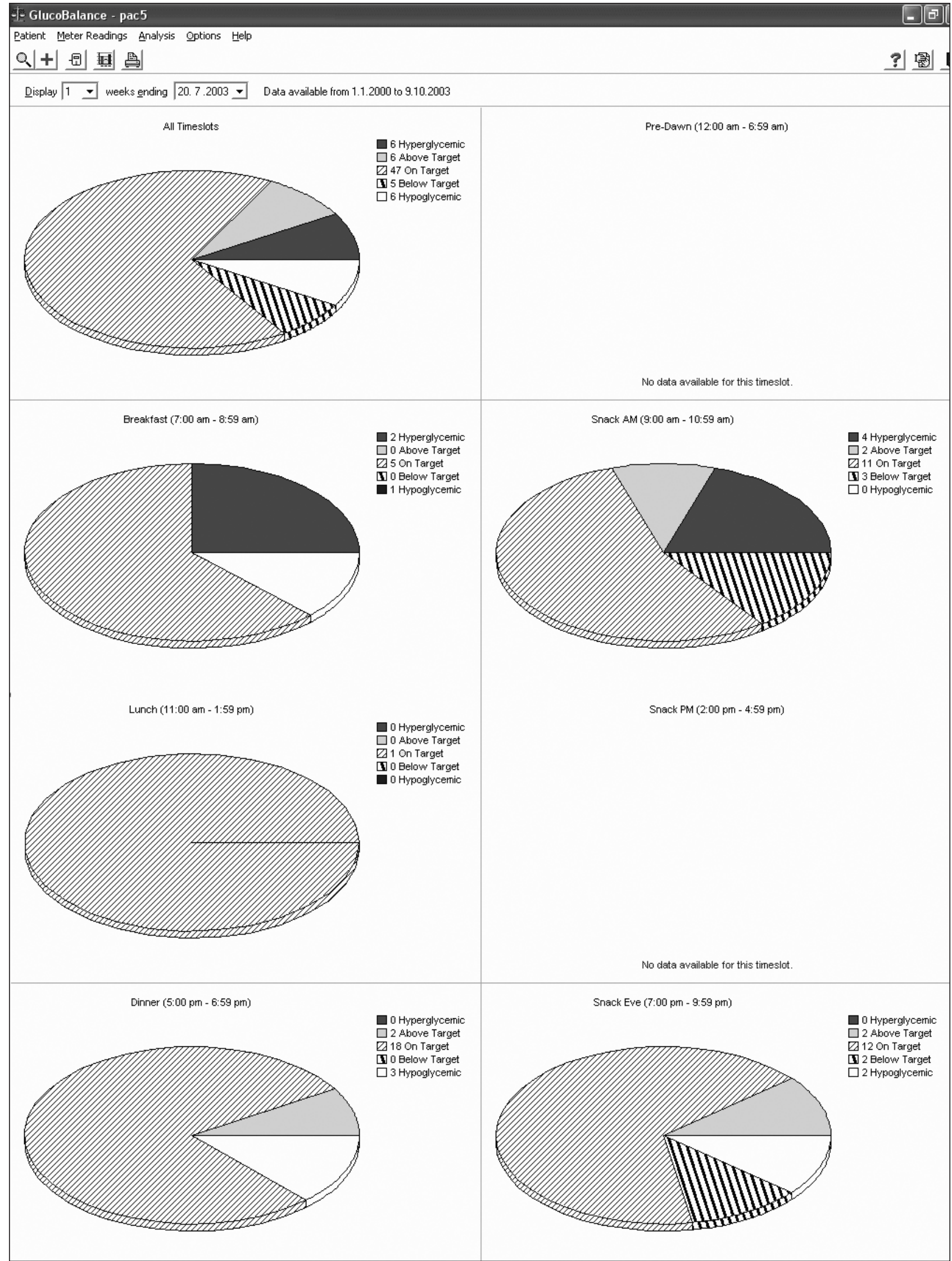

Fig. 5. Pie charts showing the frequency of B-glucose values in defined ranges in the course of defined day time periods (GSS Advance, software Glucobalance) 


\begin{tabular}{|c|c|c|c|c|c|c|c|c|c|}
\hline \multirow{2}{*}{ Display 1} & \multicolumn{9}{|c|}{ Data available from 1.1.2000 to 9.10 .2003} \\
\hline & $\begin{array}{l}\text { Pre-Dawn } \\
\text { 0:00-6:59 }\end{array}$ & $\begin{array}{l}\text { Breakfast } \\
7: 00-8: 59\end{array}$ & $\begin{array}{l}\text { Snack AM } \\
9: 00-10: 59\end{array}$ & $\begin{array}{c}\text { Lunch } \\
11: 00-13 \cdot 59\end{array}$ & $\begin{array}{c}\text { Snack PM } \\
14: 00-16: 59\end{array}$ & $\begin{array}{c}\text { Dinner } \\
17: 00-18: 59\end{array}$ & $\begin{array}{c}\text { Snack Eve } \\
19: 00-21: 59\end{array}$ & $\begin{array}{c}\text { Night } \\
\text { 22:00-23:59 }\end{array}$ & Aggregate \\
\hline \# Readings & 0 & 8 & 20 & 1 & 0 & 23 & 18 & 0 & 70 \\
\hline Maximum & & 17,37 & 21,59 & 5,16 & & 8,10 & 9,49 & & 21,59 \\
\hline 75th Percentile & & 8,30 & 8,48 & 5.16 & & 6,71 & 6,83 & & 6,96 \\
\hline Median & & 5,27 & 6,05 & 5.16 & & 6,10 & 5,69 & & 5,99 \\
\hline 25th Percentile & & 4,33 & 4,95 & 5,16 & & 4,63 & 4,58 & & 4,56 \\
\hline Minimum & & 1,05 & 3,05 & 5,16 & & 1,05 & 1,05 & & 1,05 \\
\hline Mean & & 7,28 & 7.48 & 5,16 & & 5,57 & 5,54 & & 6,30 \\
\hline Std. Dev. & & 5,59 & 4,49 & 0,00 & & 2,01 & 2,16 & & 3,47 \\
\hline \multicolumn{10}{|l|}{ Events } \\
\hline Hyper $[>=10,0]$ & & 2 & 4 & 0 & & 0 & 0 & & 6 \\
\hline Hypo $(<=3,0)$ & & 1 & 0 & 0 & & 3 & 2 & & 6 \\
\hline Above Target & & $25 \%$ & $230 \%$ & $60 \%$ & 0 & $9 \%$ & $211 \%$ & & $17 \%$ \\
\hline On Target & & $63 \%$ & $555 \%$ & 11 100\% & 1 & $78 \%$ & $1867 \%$ & & $67 \%$ \\
\hline Below Target & & $13 \%$ & $115 \%$ & $30 \%$ & 0 & $13 \%$ & $322 \%$ & & $16 \%$ \\
\hline
\end{tabular}

Fig. 6. Statistics (GSS Advance, software Glucobalance)

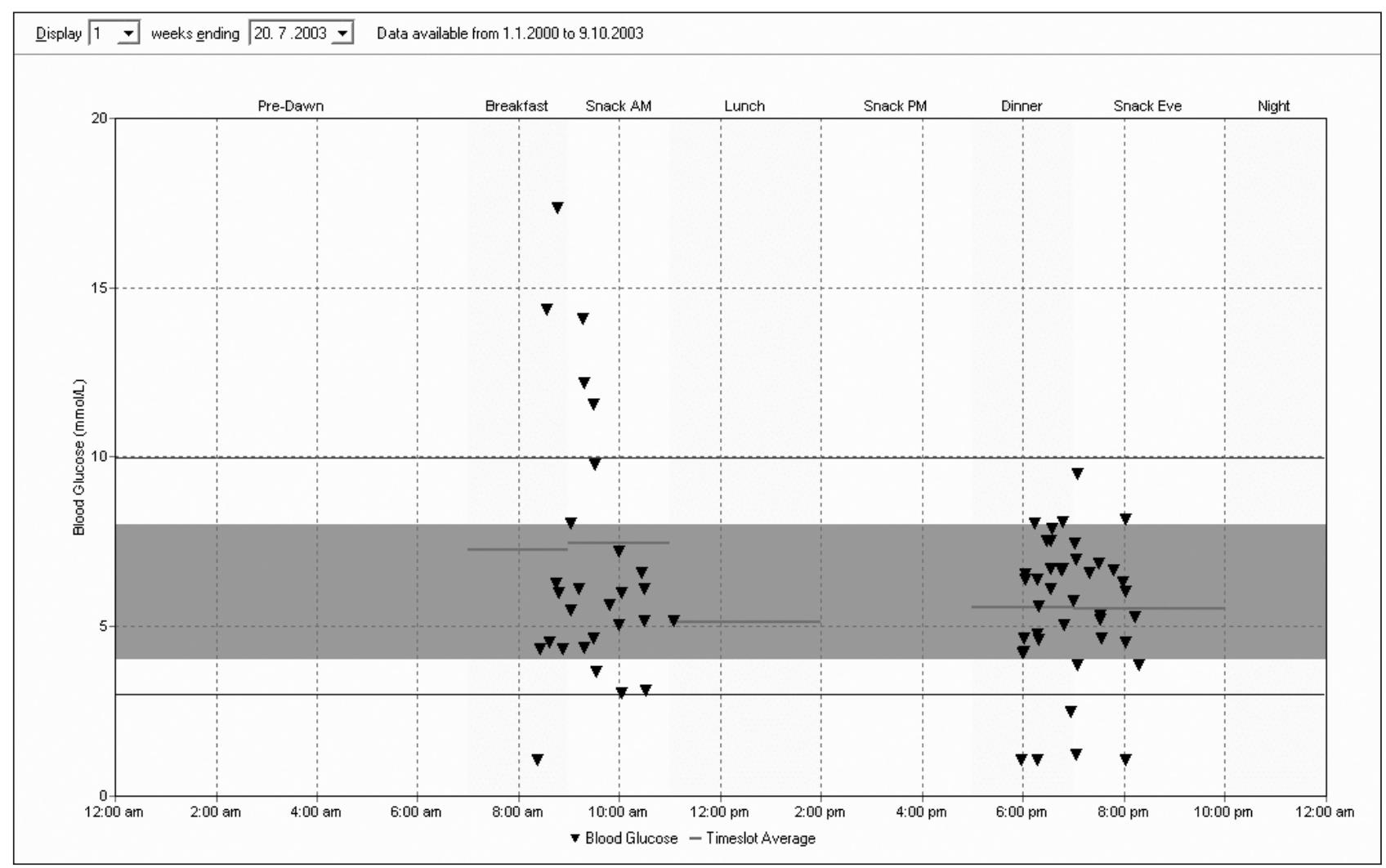

Fig. 7. Modal day: all B-glucose values measured in the course of several days drawn into the time coordinates of one day (GSS Advance, software Glucobalance) 


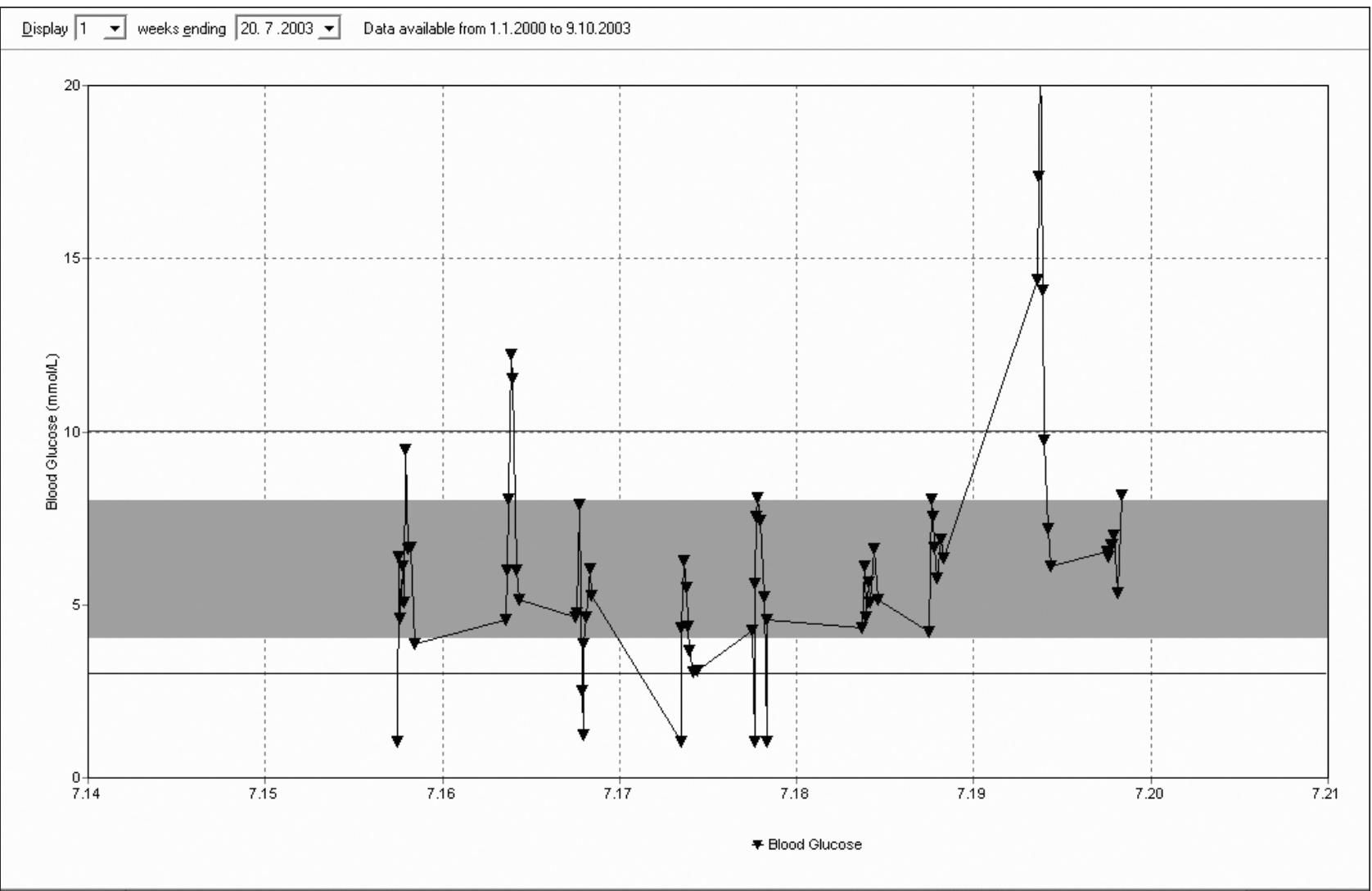

Fig. 8. Trend chart: all B-glucose values drawn in chronological order (GSS Advance, software Glucobalance)

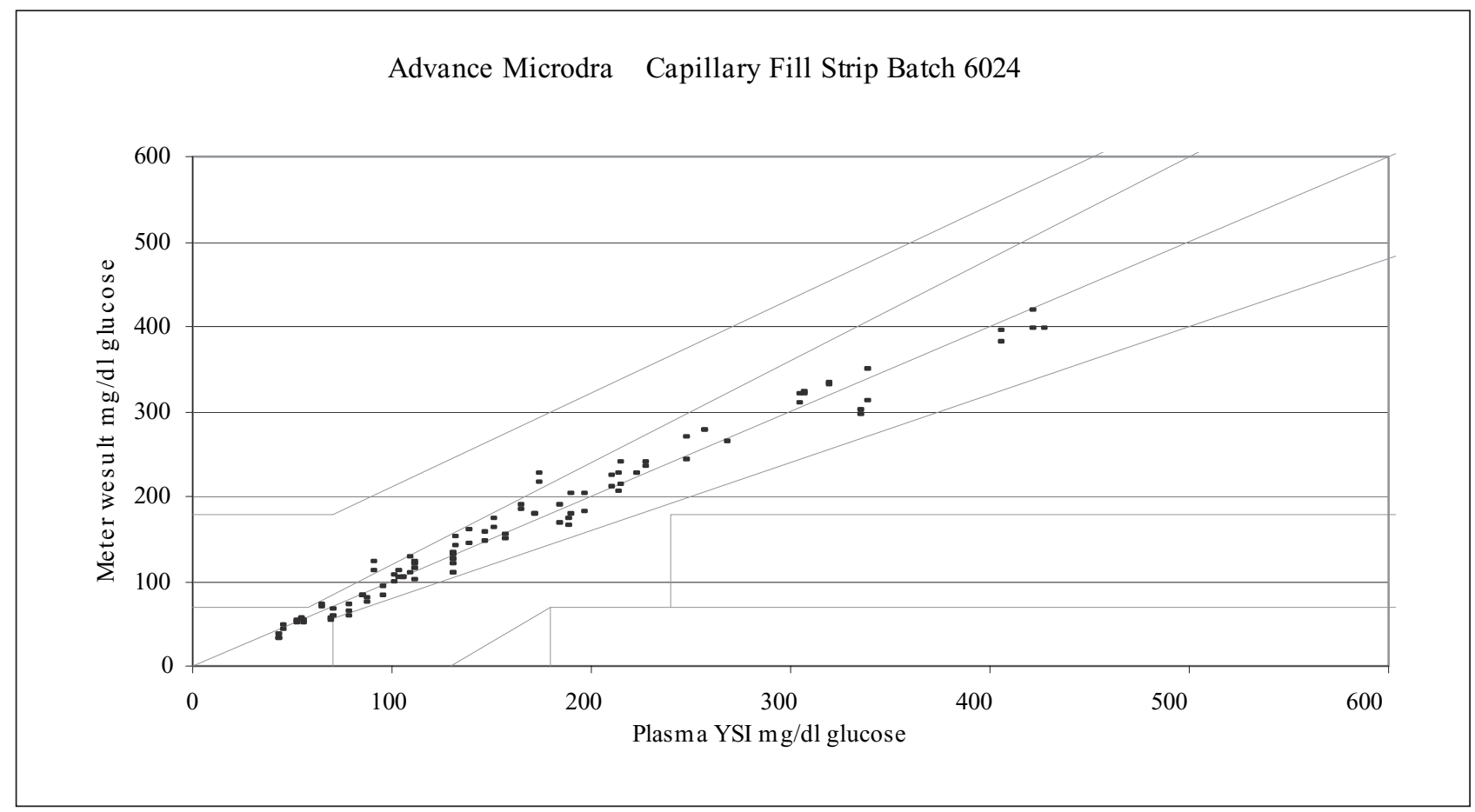

Fig. 9. Clarke Error Grid Analysis of Capillary finger-prick data from persons with type 1 diabetes; investigations were performed in Ipswitch Hospital Diabetic clinics, Great Britain (2003-2004) (ref. ${ }^{20}$ ) 


\section{DISCUSSION}

The results of B-glucose selfmonitoring depend on the quality of the glucometer, on the quality of strips and on various preanalytical and analytical factors such as temperature, humidity ${ }^{17}$, amount of blood ${ }^{19}$, B-glucose concetration and other physical-chemical parameters (including the presence of drugs etc.) influencing the activity of the glucosoxidase and the size of the developing electrical flow $6,7,8,16,18$. The registered B-glucose values collected to check the function of the glucometer system Advance was influenced by all above mentioned factors. In addition to it, the person performing the selfmonitoring is one of the inevitable parts of the whole procedure.

The laboratory regulations dealing with the accuracy of the glucometers demand that the value measured is not different from the values estimated using the approved analyzer more than by $15 \%$ (ref. ${ }^{2,5,9,10}$ ). The accuracy of both glucometers Advance and Optium has been evaluated by several researches ${ }^{1,12,14,21,23}$.

In this user-oriented study it was not possible to carry out measurements on an approved analyzer and therefore it is not possible to produce regarding evidence which kind of glucometer is more accurate. On the other hand it is possible to conclude that the average difference of B-glucose measured on glucometers Advance differs from the glucometer Optium. The magnitude of difference Advance - Optium does not seem to be dependent on the absolute B-glucose value. However, this assessment is limited, as the estimations were performed only in the $\mathrm{B} /$ glucose concentration range from 3.17 to $14.50 \mathrm{mmol} / 1$. The well known glucometer Optium was considered to be "empirically competent" to be used as a reference glucometer; nevertheless, it should be taken into consideration that the differences could be due to inaccuracy and imprecision of both glucometers, i.e., Optium and Advance ${ }^{14}$. In addition, the importance of various differences of separate pairs of glucometers is limited, because each pair was tested by a different person and under different circumstances. The outcome for the praxis is that each person should always perform selfmonitoring with the same type of the personal glucometer. Results of individual pairs of glucometers are shown (Table 1 and Table 2) to demonstrate the variability of estimations as it may be expected by the physician in daily routine.

The software Glucobalance was not used in the Czech Republic before the year 2003 and no references describing some experience with it could be found. Therefore, Fig. 4-8 was included into this paper to mention and assess its advantages and disadvantages.

In a recent Advance Micro-draw study ${ }^{20}$, the quality of the GSS was evaluated using persons with diabetes attending Ipswich Hospital Diabetic clinics from December 2003 till January 2004. Capillary blood was obtained directly from finger-pricks. Results were compared to a YSI reference analyser (Fig. 9). All data is presented without any recognised outlier removals. Very low $(<2.8 \mathrm{mmol} / \mathrm{l})$ glucose results were obtained using capillary blood that was incubated to lower glucose content prior to meter measurement. Data was evenly stratified across the blood glucose measurement range. There was no screening of patients attending the diabetic outpatients clinic. There was no limitation made on drug therapy/interferences that may have been present in the blood and no limitation on the hematocrit of any patient entering the study. (A total of 8 meters were involved in these clinical measurements).

In conclusions in may be said:

1. The results of B-glucose selfmonitoring by means of both tested glucometer systems Advance and Optium under non-standardized conditions of daily life appears to be reliable. The correlation of B-glucose measured on the glucometer Advance and Optium was strong $(\mathrm{r}=0.73)$. Glucometer Advance produce values about $0.21 \pm 0.06 \mathrm{mmol} / 1$ lower than glucometer Optium. The average difference found within each pairs of glucometers Advance - Optium varied, nevertheless, these differences are acceptable for the routine selfmonitoring.

2. The handling of glucometer Advace is not difficult for lay persons.

3. The Glucobalance software simplifies the result evaluation by each tested person. Even though there are some advantages in comparison with the PC-Link, it should be further developed.

\section{ACKNOWLEDGEMENTS}

This study was supported by research projects IGA Nr 7825-3, Ministry of Health, MSM 151100005 and Ministry of Education Youth and Sports, Czech Republic.

\section{REFERENCES}

1. Alamia V Jr, Jaekle R, Royek A, Meyer BA. (1998) Accuracy of portable glucose meters for rapid determination of amniotic fluid glukose levels. J Maternal - Fetal Med 7, 183-189.

2. American Diabetes Association. (2002) Tests of glycemia in diabetes. Diabetes Care 25, 597-599.

3. American Diabetes Association. (1994) Self-monitoring of blood glucose (Consensus Statement). Diabetes Care 17, 81-86.

4. Dillon AE, Menard MK, Rust P, Newman RB, VanDorsten JP. (1997) Glucometer analysis of one-hour glucose challenge samples. Am J Obstet Gynecol 177, 1120-1123.

5. Dohnal L, Frýdecký B, Zima T, Kalla K, Škrha J. (2000) Doporučení č. 2 České společnosti klinické biochemie ČLS JEP, Referenční laboratoře pro klinickou biochemii MZ ČR a České diabetologické společnosti: Zabezpečování jakosti měření glukometry při použití v režimu point-of-care testing. Klin Bioch Met 9/30, 78-79.

6. Dybkaer R. (1999) From total allowable error via metrological traceability to uncertainly of measurement of unbiased results. Accred Qual Assur 6, 401-405.

7. Fazel A, Koutoubi Z, Sorg TB, Mehrotra B. (1996) Influence of sample temperature on reflectance photometry and electrochemical glucometer measurements. Diabetes Care 10, 771-774. 
8. Fink KS, Christensen DB, Ellsworth A. (2002) Effect of high attitude on blood glucose meter performance. Diab Technol Therap 4, 627-635.

9. Fogh-Andersen N, D’Oratio P, Kunze K, Kuelpmann W, Mager G, Latson L. Recommendation on reporting results for blood glucose. http://www.ifcc.org/ejifcc/voll2no4/voll2no4a4.htm

10. Frýdecký B. (2001) Laboratorní diagnostika diabetu a požadavky na měření koncentrace glukózy. Klin Bioch Met 9/30, 173-177.

11. Goldstein DE, Little RR, Lorenz RA, Malone JI, Nathan D, Peterson CM. (1995) Test of glycemia in diabetes (Technical Review). Diabetes Care 18, 896-909.

12. Haag BL, Leed I. (1999) Suceptibility of two new glucose test strips to testing errors. Diabetes 48 (Suppl. 1), A415.

13. Chee F, Fernando T, van Heerden PV. (2003) Closed-loop glucose control in critically ill patients using continuous glucose monitoring systém (CGMS) in real time. IEEE Transaction on Information Technology in Biomedicine 7, 43-53.

14. Chlup R., Bartek J, Malá E, Doubravová B, Pukowietz L, Zatloukal P, Chlupová L, Zapletalová J. (2004): Uživatelská studie o správnosti a přesnosti měření glukometrů Advance, Card a Optium (Useroriented study on accuracy and precision of glucometer systems Advance, Card and Optium). Klin Bioch Metabol 12, 171-178.

15. Karter AJ, Ackerson LM, Darbinian JA, D’Agostino RB Jr, Ferrara A, Liu J, Selby JV. (2001) Self-monitoring of blood glucose levels and glycemic control: the Northern California Kaiser Permanent Diabetes Registry. Am J Med 111, 1-9.

16. Kellner A, Waldenstroem J. (1999) Estimation of total uncertainly of glucose measurements. Clin Chem Lab Med 37, Spec. Suppl. S14.
17. King JM, Eigenmann CA, Colagiuri S. (1995) Effect of ambient temperature and humidity on performance of blood glucose meters. Diab Med 12, 337-340.

18. Kurahashi K, Maruta H, Usuda Y, Ohtsuka M. (1997) Influence of blood sample oxygen tension on blood glucose concentration measured using an enzyme-electrode method (comment). Critic Care Med 25, 231-235.

19. Lewandrowaki KB, Dan L. (2002) Effects of small sample volume and interfering substances on two glucose meters (Vliv malého objemu krve v testovaném vzorku a obsahu přiměsi na dva měřici prístroje pro diabetiky). Diabetes 48 (Suppl.1), A387.

20. Payne M. (2004) Hypoguard Advance Micro-draw Performance. Klin Bioch Metabol 12, 202.

21. Puentmann I, Wosniok W, Haeckel R. (2003) Comparison of several point-of-care testing (POCT) glucometers with an established laboratory procedure for the diagnosis of type 2 diabetes using the discordance rate. A new statistical approach. Clin Chem Lab Med $41,809-820$

22. Rumley AG (1997) Improving the quality of near-patient blood glucose measurement (comment). Annals Clin Bioch 34 (Pt 3), 281-286.

23. Stahl M, Jorgensen LGM, Hyltoft Petersen P, Branslund I et al (2001) Optimization of preanalytical conditions and analysis of plasma glucose. 1. Impact of the new WHO and ADA recommendations on diagnosis of diabetes mellitus. Scand J Clin Lab Invest $61,169-180$

24. Thai AC, Ng WY, Lui KF, Yeo PP (1989) Three new glucose reflectance meters: Diascan, Glucometer II, and Reflolux II. Diabetes Res Clin Pract 7, 75-81. 